\title{
Effect of virus inducible cis-element insertion on transcription properties of improved GWSF promoter in Arabidopsis thaliana
}

\author{
Z.C. HUANG* and H. LI \\ School of Life Science and Technology, Lingnan Normal University, Zhanjiang, 524048, P.R. China
}

\begin{abstract}
An ideal synthetic promoter can accurately regulate gene expression and the minimal cauliflower mosaic virus $35 \mathrm{~S}$ promoter (GWSF) is an ideal synthetic pathogen-inducible promoter (SPIP) with several advantages. Three modified SPIPs, named as VGWSF, GWVSF, and GWSFV according to the arrangement of cis-elements, were optimized by inserting the dimer of a virus inducible $c$ is-element (TTGGGAAGGAATTTCCTACT, V-box) upstream, midstream, or downstream the GWSF sequence. The three promoters were used to replace the cauliflower mosaic virus 35S promoter in the plasmid pBI121 in order to control the expression of the $\beta$-glucuronidase (gus) gene. Transformation of Arabidopsis thaliana (ecotype Col-0) plants was performed via the Agrobacterium tumefaciens strain GV3101 by the floral dip method. The five-week-old transgenic $T_{3}$ lines were histochemically stained for GUS activity to evaluate the transcriptional properties of modified SPIPs. The VGWSF and GWVSF had low basal expressions and could not be induced by low or high temperatures and a low osmotic potential but could be induced by the tobacco mosaic virus (TMV). Although GWSFV had the highest GUS activity, it showed a substantial basal expression. After being treated with TMV, abscisic acid (ABA), salicylic acid (SA), or ethylene (Eth) for $12 \mathrm{~h}$, the expressions of modified SPIPs were evaluated by real-time quantitative PCR. With the basal expression of GWSF as a reference, each treatment was represented as $\log _{2}$ (fold to the GWSF basal level). The basal expression of VGWSF and expressions induced by TMV, ABA, SA, and Eth were 1.39, 3.42, 6.01, 4.14, and 2.26, respectively, whereas the corresponding values of GWVSF were 1.16, 4.07, 3.72, 4.65, and 3.98, respectively, and the corresponding values of GWSFV were 4.43,6.11, 4.83, 3.69, and 3.34, respectively. The results revealed that three modified SPIPs acquired virus induction activity due to the insertion of V-box. The V-box insertion position had a significant impact on transcription properties of modified SPIPs.
\end{abstract}

Additional key words: ABA, ethylene, GUS activity, SA, synthetic pathogen-inducible promoter, temperature, Tobacco mosaic virus.

Ideal pathogen-inducible promoters meet the demands of a low background expression, a wide range of inducing pathogens, rapid responses, and efficient transcriptional activities (Gurr and Rushton 2005). The promoters help to realize the desired temporal and spatial regulation of transgenes with minimal side effects. However, few native promoters have all of these advantages. Synthetic pathogen-inducible promoter (SPIP) can be used as powerful molecular tool in future biotechnology research and also in finding gene functions as the design strategies can add flexibility to control gene expression (Jameel et al. 2018). A synthetic promoter is simply a systematic combination of a minimal promoter (CaMV35S mostly) with cis-elements. The cis-elements, linked upstream to the minimal promoter, determine the expression of the target gene (Venter 2007). Synthetic promoters are usually designed by molding and adjusting the cis motifs orientation, spacing, and copy number (Dey et al. 2015). The copy number of the cis-elements modulates the inducibility and strength of a promoter (Mukherjee et al. 2004). The space between the cis-elements and the preinitiation complex has a sound effect and the space will be difficult to predict (Hernandez-Garcia and Finer 2014). In addition, permutation and combination of cis-elements has a significant impact on transcriptional properties of SPIP (Huang et al. 2017). In our opinion, transcription properties

Submitted 17 July 2019, last revision 27 January 2020, accepted 24 February 2020.

Abbreviations: ABA - abscisic acid; CaMV35S - cauliflower mosaic virus 35S; CS - connection sequence; Eth - ethylene; GUS $\beta$-glucuronidase; GWSF - minimal CaMV35S promoter; IS - insert sequence; MS - Murashige and Skoog; qPCR - quantitative PCR; SA - salicylic acid; SPIP - synthetic pathogen-inducible promoters; TMV - Tobacco mosaic virus; V-box - virus inducible cis-element. Acknowledgements: This work was funded by the National Natural Science Foundation of China (Grant No. 31570660), and the Natural Science Foundation of Guangdong Province, China (2014A030307005, 2016A030313668).

* Corresponding author; e-mail: zjyzhzc@163.com 
of promoter synthesis are difficult to predict and they need to be experimentally verified by expression analysis.

In previous studies, we designed and verified the GWSF promoter which had the advantages of low background expression, rapid response, efficient transcriptional activity, especially, responding to bacteria, fungi, salicylic acid (SA), and ethylene (Eth) (Huang et al. 2017, Huang and $\mathrm{Li}$ 2018). Herein, the GWSF was optimized by inserting V-box at a different positions. And the GWVSF with low basal expression and broad-spectrum inducible activity can potentially be further improved to apply to plant genetic engineering for disease resistance.

GWSF was composed of four pathogen-inducible ciselements (Gst1-box, W-box, S-box, and F-box) and the minimal cauliflower mosaic virus $35 \mathrm{~S}$ promoter $(-46$ to +8 TATA box). The dimer of virus inducible cis-element (TTGGGAAGGAATTTCCTACT, V-box, Kobayashi et al. 2010) was assembled with two the same ciselements interspaced with a 6 bp DNA insert sequence (IS, ACTAGA, Fig 1). Three modified SPIPs, named as VGWSF, GWVSF, and GWSFV, were optimized by inserting the dimer of V-box at upstream, midstream, or downstream of GWSF promoter. The V-box was connected with other dimers by a $10 \mathrm{bp}$ DNA connection sequence (CS, GAAGATAATC, Fig. 1). Then, the modified SPIPs were synthesized and cloned into pUC19 between Hind III and BamH I by Sangon Biotech (Shanghai, China). The modified SPIPs were used to replace the wild-type CaMV35S promoter in the plasmid pBI121 in order to control the expression of the $\beta$-glucuronidase (gus) gene. The inserted SPIP, NPT II and gus of the recombinant pBI121 plasmids were verified by restriction analysis, PCR, and partial sequencing. The primers for PCR and DNA sequencing were as follows: VGWSF forward/ reverse (5'-3'), GCAAGCTTTTGGGAAGGAAT/ TGGTGGCTGGATTATCTTCA; GWVSF forward/ reverse (5'-3'), CTGAAGATAATCCAGCCACCA/ GGATCCAGCGTGTCCTCTC; GWSFV forward/ reverse (5'-3'), CCAGAATACTAGACAGCCACCA/ GGAAGGGTCTTGCGGATTAT. NPT II gene forward/ reverse (5'-3'), GAGGCTATTCGGCTATGACTG/ ATCGGGAGCGGCGATACCGTA; and gus gene forward/reverse (5'-3'), ACACCGATACCATCAGCG/ TCACCGAAGTTCATGCCAGT. The recombinant plasmids and original vector plasmid pBI121 were transformed into Agrobacterium tumefaciens strain GV3101 as previously described (Chen et al. 1994).

Transformation of Arabidopsis thaliana (Col-0) plants was performed via A. tumefaciens strain GV3101 by the floral dip method (Zhang et al. 2006). More than thirty independent $\mathrm{T}_{0}$ plants were selected for each SPIP:gus reporter transgene by sowing seeds on a half-strength Murashige and Skoog (MS) medium with $50 \mathrm{mg} \mathrm{dm}^{-3}$ kanamycin. The five-week-old putative transgenic plants were confirmed by PCR of SPIP, NPT II, and gus as mentioned above. To determine the number of T-DNA loci, more than $3000 \mathrm{~T}_{1}$ progenies of each independent $\mathrm{T}_{0}$ line were screened for kanamycin resistance. The corresponding $\mathrm{T}_{0}$ plants with an approximately 3:1 segregation ratio of green seedlings to white seedlings in the $\mathrm{T}_{1}$ progeny were taken as single-loci transgenic plants. Five $T_{1}$ progenies of putative single-copy $\mathrm{T}_{0}$ lines were planted to harvest $\mathrm{T}_{2}$ seeds (VGWSF: lines 1, 3, 5, 6,7; GWVFS: lines 1, 3, 4, 7, 9; GWSFV: lines 1, 3, 4, 6, 8). The $\mathrm{T}_{2}$ progeny was confirmed by kanamycin resistance and PCR as mentioned above. At last, all fifteen homozygous lines were identified when the rate of green seedlings of each $\mathrm{T}_{3}$ line, selected by kanamycin, was higher than $99 \%$.

Arabidopsis thaliana $\mathrm{T}_{3}$ homozygous transgenic lines were grown in growth chambers at an irradiance of $300 \mu \mathrm{mol}$ (photon) $\mathrm{m}^{-2} \mathrm{~s}^{-1}$ during a 12 -h photoperiod, day/ night temperatures of $23 / 20{ }^{\circ} \mathrm{C}$, and a relative humidity of $70 \%$. The rosette leaves of five-week-old plants were stained for GUS activity as described Jefferson et al. (1987) to detect a basal expression histochemically. Then, individual plants were treated by high temperature, low temperature, low osmotic potential, or Tobacco mosaic virus (TMV) to valuate transcription properties of improved SPIPs. The processing conditions and methods were as follows: for high temperature treatment, plants were put in growth cabinet in which the temperature was pre-set to $37^{\circ} \mathrm{C}$ for $12 \mathrm{~h}$; for low temperature treatment, plants were put in growth cabinet in which the temperature was pre-set to $4{ }^{\circ} \mathrm{C}$ for $12 \mathrm{~h}$; for low osmotic potential treatment, plants planted in $100 \mathrm{~cm}^{3}$ of Vermiculite were irrigated with $50 \mathrm{~cm}^{3}$ of $200 \mathrm{mM} \mathrm{NaCl}$ solution for $12 \mathrm{~h}$. The diseased tobacco (Nicotiana tabacum L. cv. Xiangyan No. 3) leaves with serious symptoms of TMV were collected to extract virus particles. The leaves were ground with 3 times volume of $30 \mathrm{mM}$ phosphate buffer ( $\mathrm{pH}$ 7.2). Then, the homogenates were centrifuged at $3000 \mathrm{~g}$ and $4{ }^{\circ} \mathrm{C}$ for $5 \mathrm{~min}$. Chloroform, equivalent to one-fifth volume of leaves, was added into the supernatant to remove protein by mixing fully. After centrifuging at $4{ }^{\circ} \mathrm{C}$ and $3000 \mathrm{~g}$ for 10 min, polyethylene glycol (PEG6000) was added into the supernatant until the concentration was $6 \%(\mathrm{~m} / \mathrm{v})$. Then, $\mathrm{NaCl}$ was added until the concentration was $0.5 \mathrm{M}$. After precipitating for several hours, the precipitate was

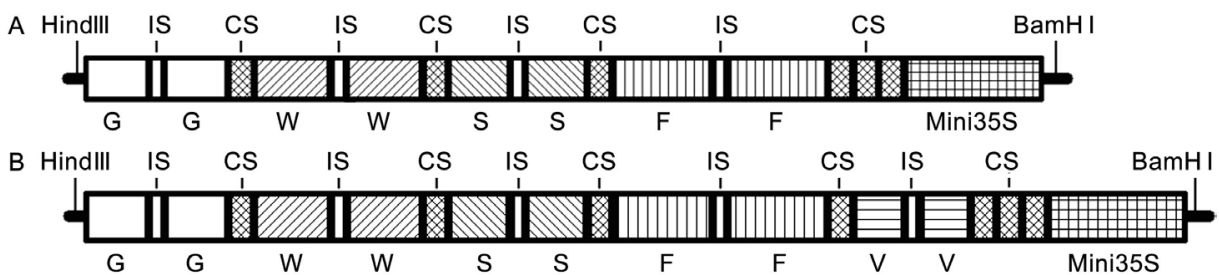

Fig. 1. A schematic diagram of synthetic pathogen-inducible promoters GWSF $(A)$ and GWSFV $(B)$. G - Gst1-box, W - W-box, S S-box, F - F-box, V - V-box, IS - 6 bp DNA insert sequence (ACTAGA), CS - 10 bp DNA connection sequence (GAAGATAATC), Mini $35 \mathrm{~S}$ - minimal CaMV 35S promoter. 


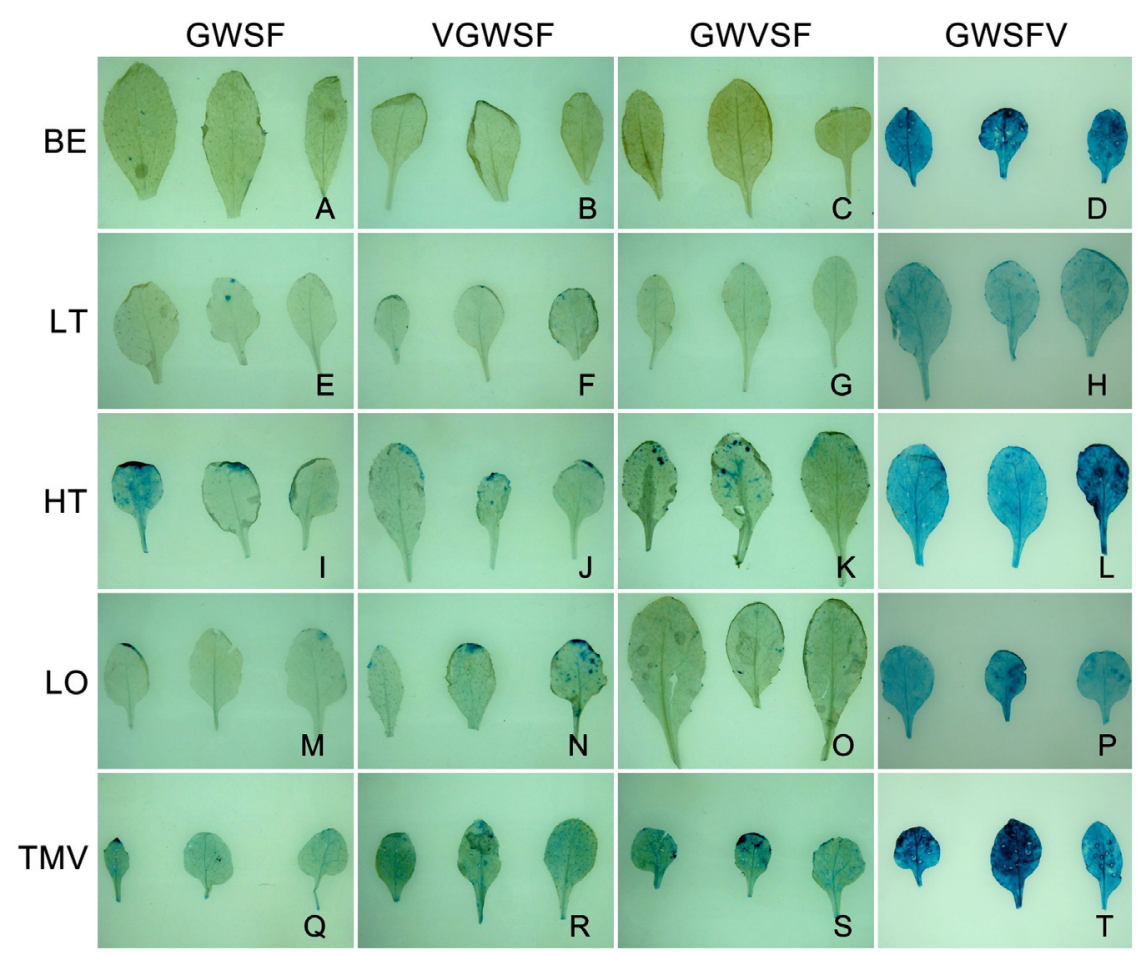

Fig. 2. Histochemical staining synthetic pathogen-inducible promoters gus transgenic Arabidopsis thaliana $\mathrm{T}_{3}$ lines treated with low temperature (LT), high temperature (HT), low osmotic potential (LO), and tobacco mosaic virus (TMV); BE - background expression.

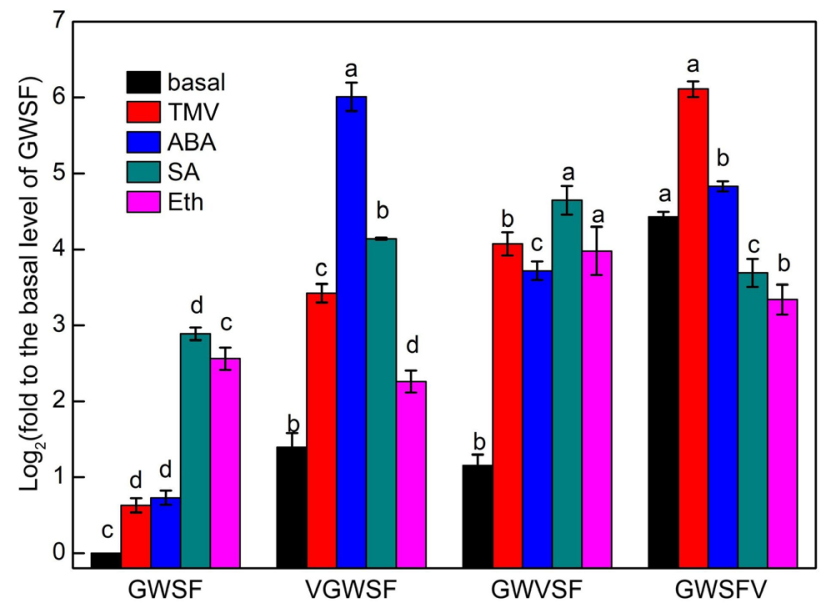

Fig. 3. Expressions of four synthetic pathogen-inducible promoters (GWSF, VGWSF, GWVSF, and GWSFV) in five-week-old transgenic Arabidopsis thaliana $\mathrm{T}_{3}$ lines after being treated with tobacco mosaic virus (TMV), abscisic acid (ABA), salicylic acid (SA) or ethylene (Eth). Basal means the basal expression. Analysis of relative $\beta$-glucuronidase (gus) expression was performed by using the $2^{-\Delta \Delta \mathrm{Ct}}$ method with the basal expression of GWSF-gus normalized to 1. The experiments were performed at least three times with similar results. Values are the means \pm SDs.

collected by centrifuging at $4{ }^{\circ} \mathrm{C}$ and $3000 \mathrm{~g}$ for $30 \mathrm{~min}$. At last, the precipitate was dissolved in $0.9 \%(\mathrm{~m} / \mathrm{v}) \mathrm{NaCl}$ and the supernatant with TMV virus particles was used as inductor after being centrifuged at $4{ }^{\circ} \mathrm{C}$ and $3000 \mathrm{~g}$ for $30 \mathrm{~min}$. Each piece of rosette leaves of five-week-old plants was inoculated with $50 \mathrm{~mm}^{3} \mathrm{TMV}$ virus particle solution by smearing or $2 \mathrm{mM}$ ABA by spraying or $75 \mu \mathrm{M}$ salicylic acid by spraying. The ethylene treatment was performed according to the method of Huang and Li (2018).

More than 15 plants of each line per treatment were used as a sample. After incubation at $25{ }^{\circ} \mathrm{C}$ for $12 \mathrm{~h}$, five pieces of leaves from five individual lines (30 to $40 \mathrm{mg}$ ) were ground in liquid nitrogen for total RNA isolation by using a UNIQ-10 Column Trizol total RNA isolation kit (Sangon Biotech). The quality and concentration of the total RNA were determined with a NanoDrop 2000C (Thermo Scientific, Waltham, MA, USA) spectrophotometer. Reverse transcription was performed according to the manufacturer's protocol (a PrimeScript RT reagent kit with gDNA eraser, TaKaRa, Dalian, China) and the cDNA was used for quantitative PCR (qPCR). The cDNA from five individual lines of the transgenic GWSF:gus lines was mixed to use as a reference to evaluate the transcription activities of SPIP. The plant materials of each treatment were in triplicates.

Transcription of the gus gene was evaluated by qPCR using a CFX 96 (Bio-Rad, Hercules, USA) and SYBR Premix Ex Taq ${ }^{\mathrm{TM}}$ II (TaKaRa). The relative expression of the gene was calculated using the EF1- $\alpha$ (AT5G60390) gene as an internal control. Primers used were: gus gene forward/reverse (5'-3'), CTGATAGCGCGTGACAAAAA/ GGCACAGCACATCAAAGAGA; EF1- $\alpha$ gene forward/ reverse (5'-3'), TGAGCACGCTCTTCTTGCTTTCA/ GGTGGTGGCATCCATCTTGTTACA. The thermal cycling conditions were as follows: initial denaturation step at $95{ }^{\circ} \mathrm{C}$ for $60 \mathrm{~s}, 40$ cycles at $94{ }^{\circ} \mathrm{C}$ for of $10 \mathrm{~s}$, at $55^{\circ} \mathrm{C}$ for $10 \mathrm{~s}$, and at $72{ }^{\circ} \mathrm{C}$ for $15 \mathrm{~s}$. The specificity of the primer pairs was verified by melting curve analysis 
from 65 to $90{ }^{\circ} \mathrm{C}$. Quantification of relative changes in gene expression was performed using the $2^{-\Delta \Delta \mathrm{Ct}}$ method as described by Livak and Schmittgen 2001 and Bio-Rad CFX Manager software v.3.1. Values from three independent experiments were calculated based on transcription of gus in GWSF: gus, which was normalized to 1.

The basal expression of GWSF was low, no obvious staining could be visible. The basal expression of GWSFV was much higher than that of GWSF when V-box was inserted downstream of GWSF. But the V-box had little effect on the background expression activity of improved promoter when it was inserted upstream or midstream of the GWSF sequence (Fig. 1). VGWSF and GWVSF had low basal expressions and could not be induced by low temperature, high temperature, and low osmotic potential. But both SPIPs could be induced by TMV. However, GWSFV had a high basal expression. Meanwhile, GWSFV could be induced by low temperature, high temperature, low osmotic potential, and TMV (Fig. 2).

After being treated $12 \mathrm{~h}$, the transcription activities in response to TMV, ABA, SA, and Eth of improved SPIPs were evaluated by qPCR. With the basal expression of GWSF as a reference, each treatment was represented as $\log _{2}$ (fold change to the GWSF basal level). The basal expression and transcription activities of VGWSF induced by TMV, ABA, SA, and Eth were 1.39, 3.42, 6.01, 4.14 and 2.26, respectively (Fig. 3). The corresponding values of GWVSF were 1.16, 4.07, 3.72, 4.65 and 3.98, respectively. The corresponding values of GWSFV were 4.43, 6.11, 4.83, 3.69 , and 3.34, respectively. The results revealed that the modified promoter was induced by TMV due to the insertion of the V-box. After the insertion of V-box, the response of the improved promoter to changes in temperature or osmotic potential was not obvious, but it significantly enhanced the response to TMV, ABA, SA, and Eth.

There are many factors that have effects on transcriptional properties of SPIP, such as cis-element function, cis-element copy number, space to TATA-box, location relative to their regulatory targets, etc. (Shokouhifar et al. 2011). At present, except for the position of V-box, all other factors were exactly the same in the constructs of the three modified SPIPs (Fig. 1). However, the basal expression of GWSFV was much higher than of VGWSF or GWVSF.

Compared with GWSF, the basal expression and expressions induced by TMV, ABA, SA, or Eth of three modified SPIPs increased significantly. Among the 3 promoters, the expression of GWSFV induced by TMV was the highest (69.1 times higher than the basal expression of GWSF); the expression of VGWSF induced by ABA was the highest (64.8 times higher than the basal expression of GWSF); and the expression of GWVSF induced by SA or Eth was the highest (25.2 or 16.2 times higher than the basal expression of GWSF, respectively). The results implied that the expressions of GWSF sharply changed after inserting the dimer of the V-box. The TMV inducible activity increased markedly due to the insertion of the V-box. The basal activity of GWSFV was much higher than that of GWSF when V-box was inserted downstream of the GWSF promoter. But the V-box had little effect on the basal expression of the modified promoter when it was inserted upstream or midstream of the GWSF sequence. The insert position of motifs had an important influence on the expressions of SPIP. It implied that the closer the inserting cis-element to CaMV-mini35S was, the more significant effect on the original sequence it caused. In summary, GWVSF had the advantages of a low background expression, efficient induction by TMV, ABA, SA, and Eth, and low induction by low or high temperature, or low osmotic potential. Our study might provide a reference for the design and subsequent improvement of the synthetic promoters.

\section{References}

Chen, H., Nelson, R.S., Sherwood, J.L.: Enhanced recovery of transformants of Agrobacterium tumefaciens after freezethaw transformation and drug selection. - Biotechniques 16: 664-668, 1994.

Dey, N., Sarkar, S., Acharya, S.: Synthetic promoters in planta. Planta 242: 1077-1094, 2015.

Gurr, S.J., Rushton, P.J.: Engineering plants with increased disease resistance: what are we going to express? - Trends Biotechnol. 23: 275-282, 2005.

Hernandez-Garcia, C.M., Finer, J.J.: Identification and validation of promoters and cis-acting regulatory elements. - Plant Sci. 217: 109-119, 2014.

Huang, Z.C., Li H.: Transcriptional properties and transcriptional activities to ethylene of a synthetic pathogen-inducible promoter GWSF in Arabidopsis thaliana. - Plant Physiol. J. 54: 121-126, 2018.

Huang, Z.C., Peng, S., Li, H., Zeng, F.H.: Transcriptional properties of eight synthetic pathogen-inducible promoters in transgenic Arabidopsis thaliana. - Biol. Plant. 61: 389-393, 2017.

Jameel, A., Muhammad, N., Wang, F.W., Liu, W.C., Zhou, Y.G., Li, H.Y., Li, X.W.: Synthetic promoters: designing the cis regulatory modules for controlled gene expression. - Mol. Biotechnol. 60: 608-620, 2018.

Jefferson, R.A., Kavanagh, T.A., Bevan, M.W.: GUS fusions: $\beta$-glucuronidase as a sensitive and versatile gene fusion marker in higher plants. - EMBO J. 6: 3901-3907, 1987.

Kobayashi, M., Ishihama, N., Yoshioka, H., Takabatake, R., Tsuda, S., Seo, S., Ohashi, Y., Mitsuhara, I.: Analyses of the cis-regulatory regions responsible for the transcriptional activation of the $\mathrm{N}$ resistance gene by tobacco mosaic virus. J. Phytopathol. 158: 826-828, 2010.

Livak, K., Schmittgen, T.D.: Analysis of relative gene expression data using real time quantitative PCR and the $2^{-\Delta \mathrm{Ct}}$ method. Methods 25: 402-408, 2001.

Mukherjee, S., Berger, M.F., Jona, G., Wang, X.S., Muzzey, D., Snyder, M., Young, R.A., Bulyk, M.L.: Rapid analysis of the DNA-binding specificities of transcription factors with DNA microarrays. - Natur. Genetics 36: 1331-1339, 2004.

Shokouhifar, F., Zamani, M.R., Motallebi, M., Mousavi, A., Malboobi, M.A.: Construction and functional analysis of pathogen-inducible synthetic promoters in Brassica napus. Biol. Plant. 55: 689-695, 2011.

Venter, M.: Synthetic promoters: genetic control through cis engineering. - Trends Plant Sci. 12: 118-124, 2007.

Zhang, X., Henriques, R., Lin, S.S., Niu, Q.W., Chua, N.H.: Agrobacterium-mediated transformation of Arabidopsis thaliana using the floral dip method. - Nat. Protocols 1: 641646, 2006. 